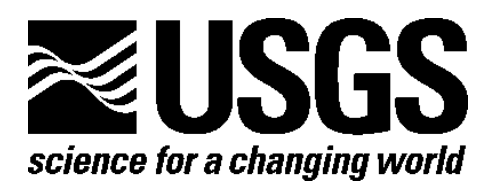

\title{
New Vitrinite Reflectance Data for the Wind River Basin, Wyoming
}

By Mark J. Pawlewicz and Thomas M. Finn

Open-File Report 2013-1002 


\section{U.S. Department of the Interior \\ KEN SALAZAR, Secretary}

\section{U.S. Geological Survey \\ Marcia K. McNutt, Director}

U.S. Geological Survey, Reston, Virginia: 2013

For more information on the USGS-the Federal source for science about the Earth,

its natural and living resources, natural hazards, and the environment-visit

http://www.usgs.gov or call 1-888-ASK-USGS

For an overview of USGS information products, including maps, imagery, and publications, visit $h$ ttp://www.usgs.gov/pubprod

To order this and other USGS information products, visit http://store.usgs.gov

Suggested citation:

Pawlewicz, M.J., and Finn, T.M., 2013, New vitrinite reflectance data for the Wind River Basin, Wyoming: U.S. Geological Survey Open-File Report 2013-1002, 11 p.

Any use of trade, product, or firm names is for descriptive purposes only and does not imply endorsement by the U.S. Government.

Although this report is in the public domain, permission must be secured from the individual copyright owners to reproduce any copyrighted material contained within this report. 


\section{Contents}

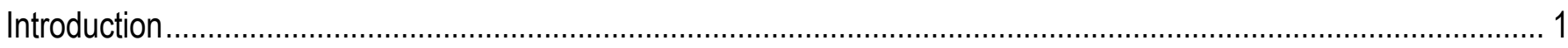

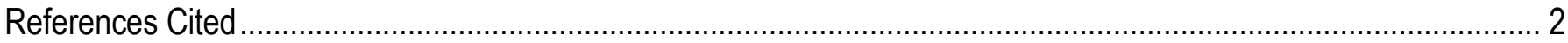

\section{Figures}

Figure 1. Map of the Rocky Mountain region showing Laramide Basins......................................................... 3

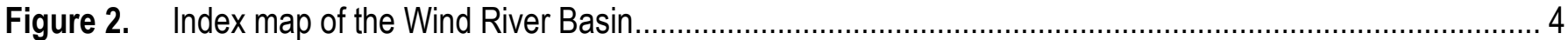

Figure 3. Stratigraphic chart of Cretaceous and Lower Tertiary rocks in the Wind River Basin ............................5 5

\section{Table}

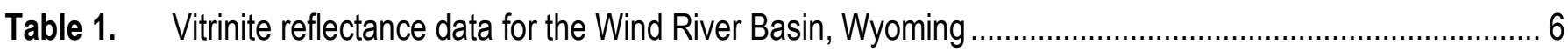




\section{New Vitrinite Reflectance Data for the Wind River Basin, Wyoming}

By Mark J. Pawlewicz and Thomas M. Finn

\section{Introduction}

The Wind River Basin is a large Laramide (Late Cretaceous through Eocene) structural and sedimentary basin that encompasses about 7,400 square miles in central Wyoming (fig. 1). The basin is bounded by the Washakie Range and Owl Creek and southern Bighorn Mountains on the north, the Casper arch on the east and northeast, and the Granite Mountains on the south, and Wind River Range on the west (figs. 1 and 2).

Important conventional and unconventional oil and gas resources have been discovered and produced from reservoirs ranging in age from Mississippian through Tertiary (Fox and Dolton, 1989; De Bruin, 1993; Johnson and others, 1996, 2007). In recent years, advances and success in horizontal drilling and multistage fracture stimulation have led to an increase in exploration and completion of wells in marine shales in other Rocky Mountain Laramide basins that were traditionally considered hydrocarbon source rocks. Important parameters that control hydrocarbon production from these shales include: reservoir thickness, amount and type of organic matter, and thermal maturity (Milici, 1993; Curtis, 2002). The purpose of this report is to present new vitrinite reflectance data collected mainly from Cretaceous marine shales in the Wind River Basin to better characterize their thermal maturity and hydrocarbon potential. These new data supplement previously published data by Johnson and others (1991), Barker and Crysdale (1993), Katz and Liro (1993), Nuccio and others (1993), Pawlewicz (1993), Nuccio and others (1996), and Finn and others (2006).

Ninety-nine samples from Cretaceous and Tertiary strata (fig. 3) were collected from well cuttings stored at the U.S. Geological Survey Core Research Center in Lakewood, Colorado. Well sample locations are shown on figure 2. All samples were analyzed for vitrinite reflectance to determine levels of thermal maturation. Preparation of samples for reflectance analysis required (1) crushing the larger pieces into 0.25 - to 1-millimeter pieces, (2) casting the pieces with epoxy in pre-cut and drilled plugs, and (3) curing the samples overnight. Subsequently, a four-step grinding and polishing process was implemented that included sanding with progressively finer sandpaper (60 to 600 grit) followed by a two-step polishing process ( 0.3 and 0.05 micron). Vitrinite reflectance measurements were determined at $500 \mathrm{X}$ magnification using plane-polarized incident white light and a 546-nanometer monochromatic filter in immersion oil. For samples containing sufficiently high quality vitrinite, at least 25 measurements were recorded. For samples of poorer quality, either due to a poor polish or to the presence of mineral or other inorganic material, fewer measurements were recorded. Analytical results are given in table 1. 


\section{References Cited}

Barker, C.E., and Crysdale, B.L., 1993, Burial and temperature history of gas generation from coaly organic matter in the Late Cretaceous Mesaverde Formation and associated rocks in the deeper portions of the Wind River Basin, Wyoming, in Stroock, Betty, and Andrew, Sam, eds., Jubilee Anniversary $44^{\text {th }}$ Field Conference: Wyoming Geological Association, Guidebook, p. 235-258.

Curtis, J.B., 2002, Fractured shale-gas systems: American Association of Petroleum Geologists Bulletin, v. 86 , no. 11 , p. 1,921-1,938.

De Bruin, R.H., 1993, Overview of oil and gas geology of Wyoming, in Snoke, A.W., Steidtmann, J.R., and Roberts, S.M., eds., Geology of Wyoming: Geological Survey of Wyoming Memoir No. 5, p. 836-873.

Dickinson, W.R., Klute, M.A., Hayes, M.J., Janecke, S.U., Lundin, E.R., McKittrick, M.A., and Olivares, M.D., 1988, Paleographic and paleotectonic setting of Laramide sedimentary basins in the central Rocky Mountain region: Geological Society of America Bulletin, v. 100, p. 1,023-1,039.

Finn, T.M., Roberts, L.N.R., and Pawlewicz, M.J., 2006, Vitrinite reflectance data for the Wind River Basin, central Wyoming: U.S. Geological Survey Open-File Report 2006-1015, 4 p.

Fox, J.E., and Dolton, G.L., 1989, Petroleum geology of the Wind River and Bighorn Basins, Wyoming and Montana: U.S. Geological Survey Open-File Report 87-450P, 41 p.

Green, G.N., and Drouillard, P.H., 1994, The digital geologic map of Wyoming in ARC/INFO format:

U.S. Geological Survey Open-File Report 94-0425, scale 1:500,000.

Johnson, R.C., Finn, T.M., Crovelli, R.A., and Balay, R.H., 1996, An assessment of in-place gas resources in low-permeability Upper Cretaceous and Lower Tertiary sandstone reservoirs, Wind River Basin, Wyoming: U.S. Geological Survey Open-File Report 96-264, 67 p.

Johnson, R.C., Barker, C.E., Pawlewicz, M.J., Crysdale, B.L., Clark, A.C., and Rice, D.D., 1991, Preliminary results of coalbed methane assessment of the Wind River Indian Reservation, Wyoming, in Schwochow, S.D., Murray, D.K., and Fahy, M.F., eds., Coalbed methane of western North America: Rocky Mountain Association of Geologists, p. 273-284.

Johnson, R.C., Finn, T.M., Kirschbaum, M.A., Roberts, S.B., Roberts, L.N.R., Cook, T., and Taylor, D.J., 2007, The Cretaceous-Lower Tertiary Composite Total Petroleum System, Wind River Basin, Wyoming, Petroleum Systems and Geologic Assessment of Oil and Gas Resources in the Wind River Basin Province, Wyoming: U.S. Geological Survey Digital Data Series DDS-69-J, chap. 4, 96 p., CD-ROM.

Katz, B.J., and Liro, L.M., 1993, The Waltman Shale Member, Fort Union Formation, Wind River Basin-A Paleocene clastic lacustrine source system, in Keefer, W.R., Metzger, W.J., and Godwin, L.H., eds., Oil and gas and other resources of the Wind River Basin: Wyoming Geological Association Special Symposium, p. 163-174.

Milici, R.C., 1993, Autogenic gas (self sourced) from shales-An example from the Appalachian Basin: U.S. Geological Professional Paper 1570, p. 253-278.

Nuccio, V.F., Finn, T.M., and Pawlewicz, M.J., 1993, Surface vitrinite reflectance study of the Wind River Basin, central Wyoming, in Keefer, W.R., Metzger, W.J., and Godwin, L.H., eds., Oil and gas and other resources of the Wind River Basin: Wyoming Geological Association Special Symposium, p. 307-317.

Nuccio, V.F., Finn, T.M., and Johnson, R.C., 1996, Thermal maturity data for the assessment of gas resources in the Wind River Basin, Wyoming: U.S. Geological Survey Open-File Report 96-064, 57 p.

Pawlewicz, M.J., 1993, Vitrinite reflectance and geothermal gradients in the Wind River Basin, in Keefer, W.R., Metzger, W.J., and Godwin, L.H., eds., Oil and gas and other resources of the Wind River Basin: Wyoming Geological Association Special Symposium, p. 295-306. 


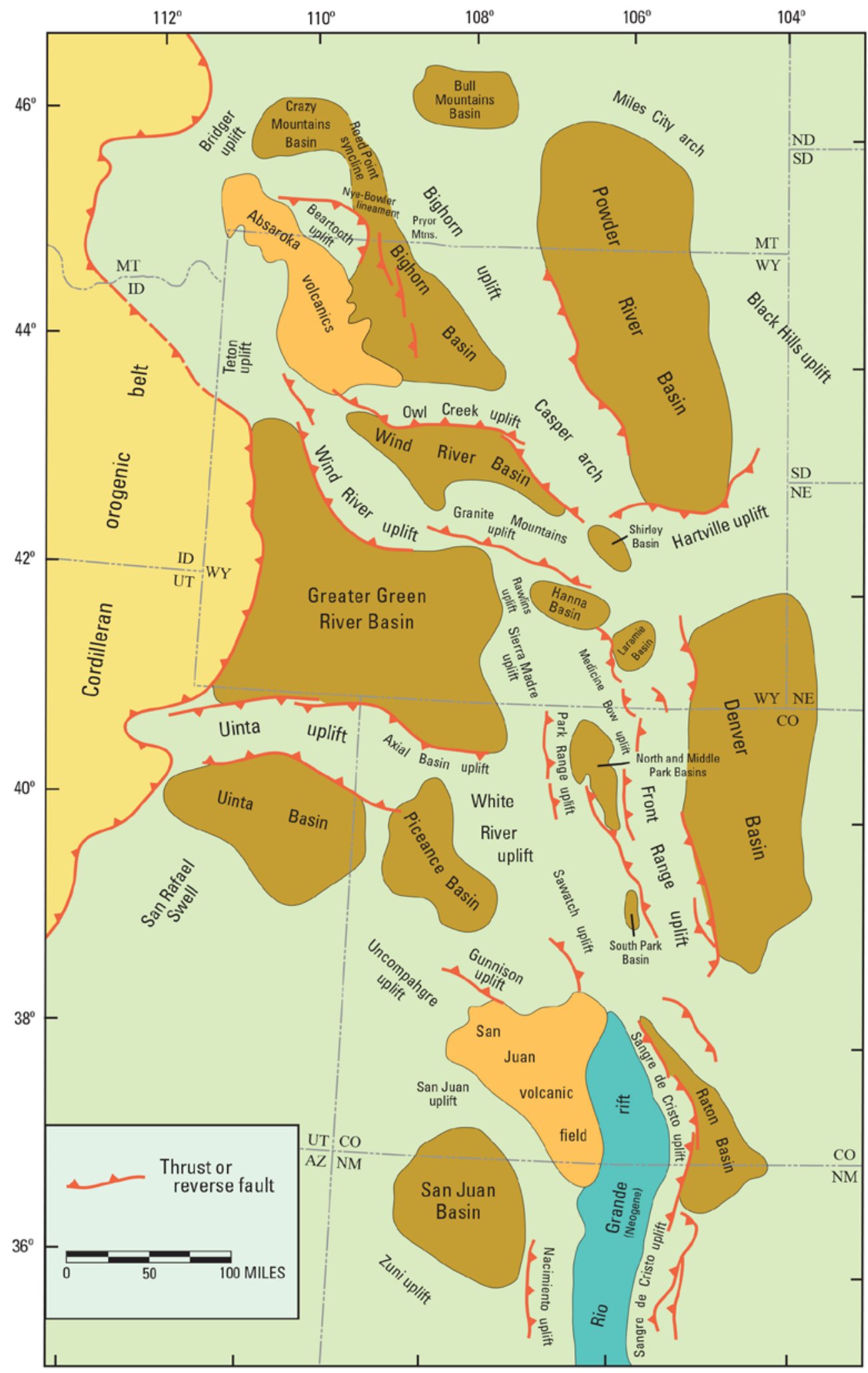

Figure 1. Map of the Rocky Mountain region extending from southern Montana to northern New Mexico showing locations of Laramide sedimentary and structural basins and related features. Modified from Dickinson and others (1988). 


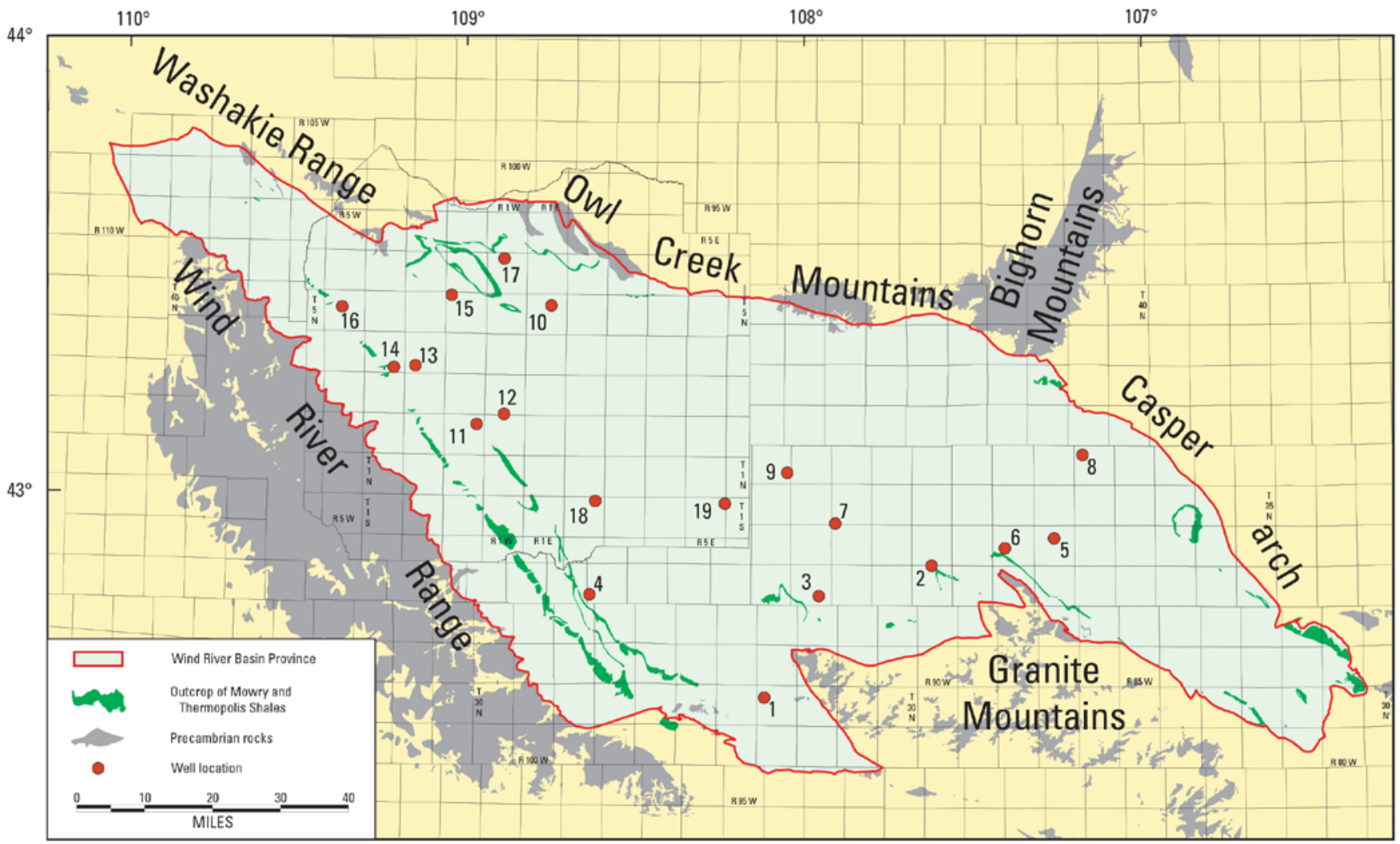

Figure 2. Map showing general outline of the Wind River Basin, bordering mountain ranges, distribution of major rock units, and sample localities. Outcrops from Green and Drouillard (1994). Province boundary from Johnson and others (2007). Numbers refer to column 1 (map no.) on table 1. 


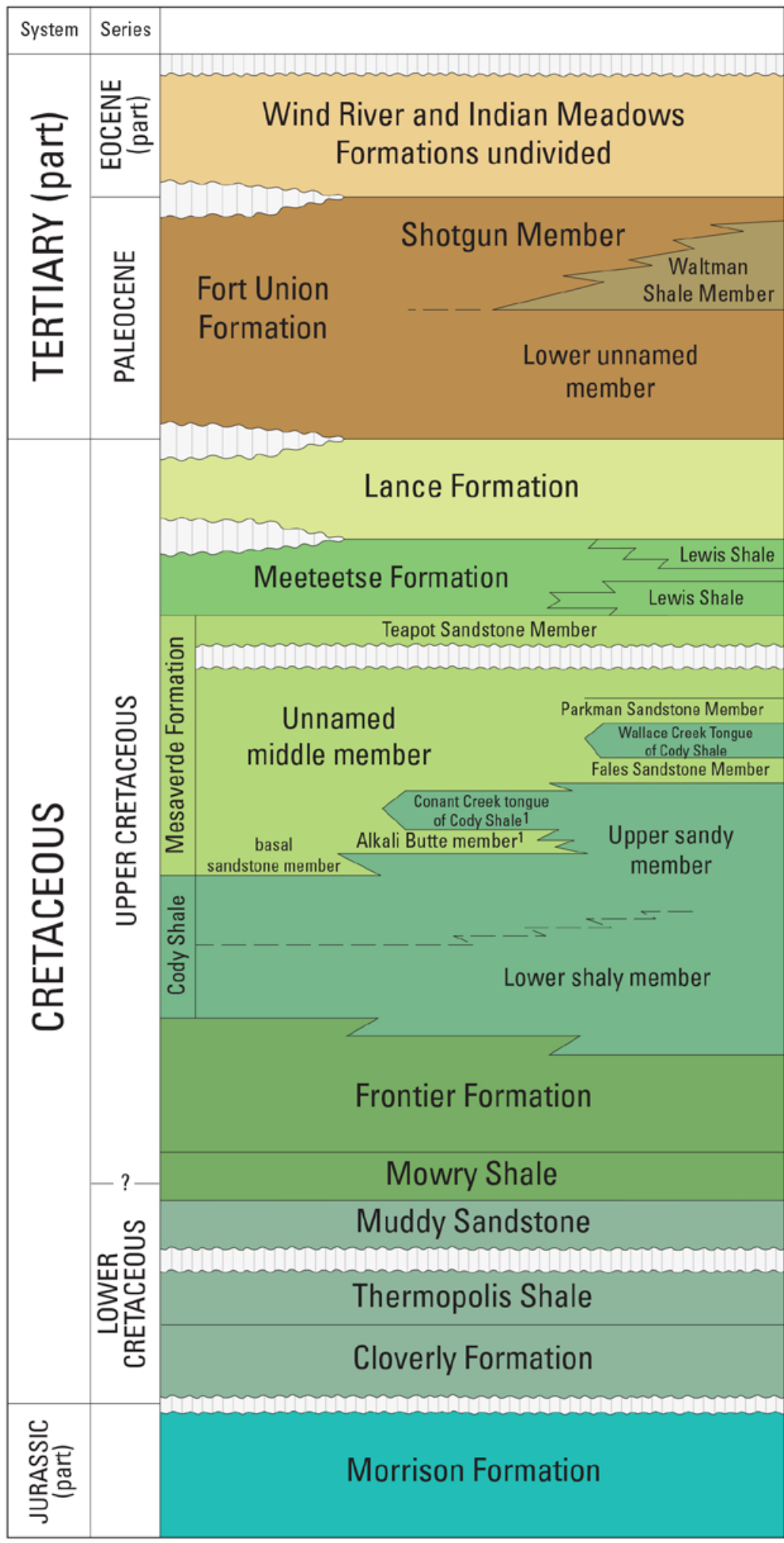

$1_{\text {informal units }}$

Figure 3. Generalized stratigraphic chart of Upper Jurassic, Cretaceous, and lower Tertiary rocks in the Wind River Basin. 
Table 1. Vitrinite reflectance data and locations for cuttings samples from wells drilled for oil and gas exploration and production in the Wind River Basin, Wyoming. Map no. refers to sample location numbers shown on figure 2.

[API, well number assigned by American Petroleum Institute; Tnsp., township; Rng., range; Sec., section; Lith., lithology; \%Ro, vitrinite reflectance measurement; $n$, number of readings per sample; nr, no reading. Depth is in feet.]

\begin{tabular}{|c|c|c|c|c|c|c|c|c|c|c|c|c|}
\hline $\begin{array}{c}\text { Map } \\
\text { no. }\end{array}$ & API & Tnsp. & Rng. & Sec. & Operator & Well & $\begin{array}{c}\text { Top } \\
\text { depth }\end{array}$ & $\begin{array}{c}\text { Bottom } \\
\text { depth }\end{array}$ & Formation & Lith. & $\% R_{0}$ & $\mathrm{n}$ \\
\hline 1 & 49013202250000 & $30 N$ & $94 \mathrm{~W}$ & 8 & Oil \& Gas Futures & Reserve-Govt. 1 & 2,000 & 2,010 & Mowry & shale & 0.94 & 12 \\
\hline 1 & 49013202250000 & $30 \mathrm{~N}$ & $94 \mathrm{~W}$ & 8 & Oil \& Gas Futures & Reserve-Govt. 1 & 2,200 & 2,210 & Mowry & shale & 0.89 & 12 \\
\hline 1 & 49013202250000 & $30 \mathrm{~N}$ & $94 \mathrm{~W}$ & 8 & Oil \& Gas Futures & Reserve-Govt. 1 & 2,330 & 2,340 & Thermopolis & shale & 0.99 & 17 \\
\hline 2 & 49013057600000 & $33 N$ & $90 \mathrm{~W}$ & 4 & Superior Oil & Govt.-Davis 81-4 & 2,060 & 2,070 & Frontier & shale & 0.57 & 5 \\
\hline 2 & 49013057600000 & $33 N$ & $90 \mathrm{~W}$ & 4 & Superior Oil & Govt.-Davis 81-4 & 2,740 & 2,770 & Frontier & shale & 0.82 & 11 \\
\hline 2 & 49013057600000 & $33 N$ & $90 \mathrm{~W}$ & 4 & Superior Oil & Govt.-Davis 81-4 & 3,000 & 3,010 & Mowry & shale & 0.61 & 9 \\
\hline 2 & 49013057600000 & $33 N$ & $90 \mathrm{~W}$ & 4 & Superior Oil & Govt.-Davis 81-4 & 3,260 & 3,280 & Mowry & shale & 0.62 & 6 \\
\hline 2 & 49013057600000 & $33 \mathrm{~N}$ & $90 \mathrm{~W}$ & 4 & Superior Oil & Govt.-Davis 81-4 & 3,400 & 3,410 & Thermopolis & shale & 1.30 & 9 \\
\hline 3 & 49013204640000 & $33 \mathrm{~N}$ & $93 \mathrm{~W}$ & 26 & Oil Resources & Govt-Curry 26-12 & 2,230 & 2,260 & Frontier & shale & 0.63 & 15 \\
\hline 3 & 49013204640000 & $33 N$ & $93 \mathrm{~W}$ & 26 & Oil Resources & Govt-Curry 26-12 & 2,500 & 2,510 & Mowry & shale & 0.82 & 7 \\
\hline 3 & 49013204640000 & $33 N$ & $93 \mathrm{~W}$ & 26 & Oil Resources & Govt-Curry 26-12 & 2,650 & 2,660 & Mowry & shale & 0.81 & 8 \\
\hline 3 & 49013204640000 & $33 N$ & $93 \mathrm{~W}$ & 26 & Oil Resources & Govt-Curry 26-12 & 2,760 & 2,770 & Thermopolis & shale & 0.69 & 10 \\
\hline 4 & 49013211920000 & $33 \mathrm{~N}$ & $98 \mathrm{~W}$ & 30 & R. Klabzuba & 1-30 Federal & 1,770 & 1,780 & Mowry & shale & 0.99 & 16 \\
\hline 4 & 49013211920000 & $33 N$ & $98 \mathrm{~W}$ & 30 & R. Klabzuba & 1-30 Federal & 2,000 & 2,020 & Mowry & shale & 0.70 & 6 \\
\hline 4 & 49013211920000 & $33 N$ & $98 \mathrm{~W}$ & 30 & R. Klabzuba & 1-30 Federal & 2,200 & 2,210 & Thermopolis & shale & 0.96 & 17 \\
\hline 4 & 49013211920000 & $33 N$ & $98 \mathrm{~W}$ & 30 & R. Klabzuba & 1-30 Federal & 2,340 & 2,350 & Cloverly & shale & 1.24 & 10 \\
\hline
\end{tabular}


Table 1. Vitrinite reflectance data and locations for cuttings samples from wells drilled for oil and gas exploration and production in the Wind River Basin, Wyoming. Map no. refers to sample location numbers shown on figure 2.-Continued

[API, well number assigned by American Petroleum Institute; Tnsp., township; Rng., range; Sec., section; Lith., lithology; \%Ro, vitrinite reflectance measurement; $n$, number of readings per sample; $\mathrm{nr}$, no reading. Depth is in feet.]

\begin{tabular}{|c|c|c|c|c|c|c|c|c|c|c|c|c|}
\hline $\begin{array}{c}\text { Map } \\
\text { no. }\end{array}$ & API & Tnsp. & Rng. & Sec. & Operator & Well & $\begin{array}{c}\text { Top } \\
\text { depth }\end{array}$ & $\begin{array}{c}\text { Bottom } \\
\text { depth }\end{array}$ & Formation & Lith. & $\% R_{0}$ & $n$ \\
\hline 5 & 49025210670000 & $34 \mathrm{~N}$ & $87 \mathrm{~W}$ & 9 & Davis Oil & 1 RK Federal & 4,680 & 4,710 & Mesaverde & coal & 0.46 & 25 \\
\hline 5 & 49025210670000 & $34 \mathrm{~N}$ & $87 \mathrm{~W}$ & 9 & Davis Oil & 1 RK Federal & 7,310 & 7,320 & Cody & shale & 0.82 & 10 \\
\hline 5 & 49025210670000 & $34 \mathrm{~N}$ & $87 \mathrm{~W}$ & 9 & Davis Oil & 1 RK Federal & 7,660 & 7,670 & Cody & shale & 0.53 & 11 \\
\hline 5 & 49025210670000 & $34 \mathrm{~N}$ & $87 \mathrm{~W}$ & 9 & Davis Oil & 1 RK Federal & 9,530 & 9,540 & Cody & shale & 0.83 & 11 \\
\hline 5 & 49025210670000 & $34 \mathrm{~N}$ & $87 \mathrm{~W}$ & 9 & Davis Oil & 1 RK Federal & 10,310 & 10,340 & Frontier & shale & 0.62 & 11 \\
\hline 5 & 49025210670000 & $34 \mathrm{~N}$ & $87 \mathrm{~W}$ & 9 & Davis Oil & 1 RK Federal & 10,690 & 10,700 & Mowry & shale & 0.73 & 13 \\
\hline 5 & 49025210670000 & $34 \mathrm{~N}$ & $87 \mathrm{~W}$ & 9 & Davis Oil & 1 RK Federal & 10,820 & 10,830 & Thermopolis & shale & 0.53 & 7 \\
\hline 6 & 49025058260000 & $34 \mathrm{~N}$ & $88 \mathrm{~W}$ & 20 & M.K.M Exploration & 1 Wolters-Fed. & 1,560 & 1,570 & Mowry & shale & 0.64 & 12 \\
\hline 6 & 49025058260000 & $34 \mathrm{~N}$ & $88 \mathrm{~W}$ & 20 & M.K.M Exploration & 1 Wolters-Fed. & 1,900 & 1,920 & Mowry & shale & 0.55 & 9 \\
\hline 6 & 49025058260000 & $34 \mathrm{~N}$ & $88 \mathrm{~W}$ & 20 & M.K.M Exploration & 1 Wolters-Fed. & 2,020 & 2,030 & Thermopolis & shale & 0.70 & 11 \\
\hline 7 & 49013069220000 & $35 \mathrm{~N}$ & $92 \mathrm{~W}$ & 31 & Sun Oil Company & Wolf-Federal 1 & 1,880 & 1,900 & Fort Union & coal & 0.68 & 19 \\
\hline 7 & 49013069220000 & $35 \mathrm{~N}$ & $92 \mathrm{~W}$ & 31 & Sun Oil Company & Wolf-Federal 1 & 3,040 & 3,060 & Meeteetse & coal & 0.54 & 11 \\
\hline 7 & 49013069220000 & $35 \mathrm{~N}$ & $92 \mathrm{~W}$ & 31 & Sun Oil Company & Wolf-Federal 1 & 3,790 & 3,810 & Mesaverde & coal & 0.56 & 27 \\
\hline 7 & 49013069220000 & $35 \mathrm{~N}$ & $92 \mathrm{~W}$ & 31 & Sun Oil Company & Wolf-Federal 1 & 5,030 & 5,060 & Mesaverde & coal & 0.54 & 13 \\
\hline 7 & 49013069220000 & $35 \mathrm{~N}$ & $92 \mathrm{~W}$ & 31 & Sun Oil Company & Wolf-Federal 1 & 8,970 & 8,980 & Cody & shale & 0.92 & 17 \\
\hline 7 & 49013069220000 & $35 \mathrm{~N}$ & $92 \mathrm{~W}$ & 31 & Sun Oil Company & Wolf-Federal 1 & 9,700 & 9,710 & Frontier & shale & 1.08 & 12 \\
\hline 7 & 49013069220000 & $35 \mathrm{~N}$ & $92 \mathrm{~W}$ & 31 & Sun Oil Company & Wolf-Federal 1 & 9,900 & 9,910 & Mowry & shale & 0.93 & 10 \\
\hline 7 & 49013069220000 & $35 \mathrm{~N}$ & $92 \mathrm{~W}$ & 31 & Sun Oil Company & Wolf-Federal 1 & 10,060 & 10,070 & Mowry & shale & 1.11 & 9 \\
\hline 7 & 49013069220000 & $35 \mathrm{~N}$ & $92 \mathrm{~W}$ & 31 & Sun Oil Company & Wolf-Federal 1 & 10,190 & 10,200 & Thermopolis & shale & 1.10 & 12 \\
\hline
\end{tabular}


Table 1. Vitrinite reflectance data and locations for cuttings samples from wells drilled for oil and gas exploration and production in the Wind River Basin, Wyoming. Map no. refers to sample location numbers shown on figure 2.-Continued

[API, well number assigned by American Petroleum Institute; Tnsp., township; Rng., range; Sec., section; Lith., lithology; \%Ro, vitrinite reflectance measurement; $n$, number of readings per sample; $n r$, no reading. Depth is in feet.]

\begin{tabular}{|c|c|c|c|c|c|c|c|c|c|c|c|c|}
\hline $\begin{array}{l}\text { Map } \\
\text { no. }\end{array}$ & API & Tnsp. & Rng. & Sec. & Operator & Well & $\begin{array}{l}\text { Top } \\
\text { depth }\end{array}$ & $\begin{array}{c}\text { Bottom } \\
\text { depth }\end{array}$ & Formation & Lith. & $\% R_{0}$ & $\mathrm{n}$ \\
\hline 8 & 49025217680000 & $36 \mathrm{~N}$ & $86 \mathrm{~W}$ & 8 & Coastal Oil \& Gas & Bullfrog 3-8-36-86 & 12,680 & 12,710 & Mesaverde & coal & 0.57 & 7 \\
\hline 8 & 49025217680000 & $36 \mathrm{~N}$ & $86 \mathrm{~W}$ & 8 & Coastal Oil \& Gas & Bullfrog 3-8-36-86 & 13,640 & 13,650 & Cody & shale & 1.18 & 12 \\
\hline 8 & 49025217680000 & $36 \mathrm{~N}$ & $86 \mathrm{~W}$ & 8 & Coastal Oil \& Gas & Bullfrog 3-8-36-86 & 15,000 & 15,010 & Cody & shale & 1.24 & 9 \\
\hline 8 & 49025217680000 & $36 \mathrm{~N}$ & $86 \mathrm{~W}$ & 8 & Coastal Oil \& Gas & Bullfrog 3-8-36-86 & 16,000 & 16,020 & Cody & shale & 1.54 & 7 \\
\hline 8 & 49025217680000 & $36 \mathrm{~N}$ & $86 \mathrm{~W}$ & 8 & Coastal Oil \& Gas & Bullfrog 3-8-36-86 & 17,140 & 17,160 & Cody & shale & 1.46 & 8 \\
\hline 8 & 49025217680000 & $36 \mathrm{~N}$ & $86 \mathrm{~W}$ & 8 & Coastal Oil \& Gas & Bullfrog 3-8-36-86 & 18,230 & 18,250 & Frontier & shale & 1.74 & 7 \\
\hline 8 & 49025217680000 & $36 \mathrm{~N}$ & $86 \mathrm{~W}$ & 8 & Coastal Oil \& Gas & Bullfrog 3-8-36-86 & 19,310 & 19,320 & Frontier & shale & $\mathrm{nr}$ & \\
\hline 8 & 49025217680000 & $36 \mathrm{~N}$ & $86 \mathrm{~W}$ & 8 & Coastal Oil \& Gas & Bullfrog 3-8-36-86 & 19,450 & 19,460 & Mowry & shale & 2.01 & 9 \\
\hline 8 & 49025217680000 & $36 \mathrm{~N}$ & $86 \mathrm{~W}$ & 8 & Coastal Oil \& Gas & Bullfrog 3-8-36-86 & 19,640 & 19,650 & Mowry & shale & 2.41 & 10 \\
\hline 8 & 49025217680000 & $36 \mathrm{~N}$ & $86 \mathrm{~W}$ & 8 & Coastal Oil \& Gas & Bullfrog 3-8-36-86 & 19,800 & 19,820 & Thermopolis & shale & 2.19 & 10 \\
\hline 9 & 49013205650000 & $36 \mathrm{~N}$ & $94 \mathrm{~W}$ & 25 & Pan Canadian & Fuller Reservoir II & 10,800 & 10,810 & Cody & shale & 1.33 & 13 \\
\hline 9 & 49013205650000 & $36 \mathrm{~N}$ & $94 \mathrm{~W}$ & 25 & Pan Canadian & Fuller Reservoir II & 12,000 & 12,040 & Cody & shale & 1.23 & 8 \\
\hline 9 & 49013205650000 & $36 \mathrm{~N}$ & $94 \mathrm{~W}$ & 25 & Pan Canadian & Fuller Reservoir II & 13,310 & 13,320 & Cody & shale & 1.52 & 17 \\
\hline 9 & 49013205650000 & $36 \mathrm{~N}$ & $94 \mathrm{~W}$ & 25 & Pan Canadian & Fuller Reservoir II & 13,850 & 13,870 & Frontier & shale & 1.43 & 13 \\
\hline 9 & 49013205650000 & $36 \mathrm{~N}$ & $94 \mathrm{~W}$ & 25 & Pan Canadian & Fuller Reservoir II & 14,130 & 14,140 & Frontier & shale & 1.68 & 15 \\
\hline 9 & 49013205650000 & $36 \mathrm{~N}$ & $94 \mathrm{~W}$ & 25 & Pan Canadian & Fuller Reservoir II & 14,350 & 14,360 & Mowry & shale & 1.44 & 11 \\
\hline 9 & 49013205650000 & $36 \mathrm{~N}$ & $94 \mathrm{~W}$ & 25 & Pan Canadian & Fuller Reservoir II & 14,560 & 14,570 & Mowry & shale & 1.72 & 12 \\
\hline 9 & 49013205650000 & $36 \mathrm{~N}$ & $94 \mathrm{~W}$ & 25 & Pan Canadian & Fuller Reservoir II & 14,650 & 14,670 & Thermopolis & shale & 1.70 & 17 \\
\hline 10 & 49013204400000 & $5 \mathrm{~N}$ & $1 \mathrm{E}$ & 10 & Continental Oil & Tribal 10-1 & 7,100 & 7,110 & Cody & shale & 0.68 & 11 \\
\hline 10 & 49013204400000 & $5 \mathrm{~N}$ & $1 \mathrm{E}$ & 10 & Continental Oil & Tribal 10-1 & 8,300 & 8,310 & Mowry & shale & 0.73 & 13 \\
\hline 10 & 49013204400000 & $5 \mathrm{~N}$ & $1 \mathrm{E}$ & 10 & Continental Oil & Tribal 10-1 & 8,500 & 8,510 & Mowry & shale & 0.72 & 15 \\
\hline 10 & 49013204400000 & $5 \mathrm{~N}$ & $1 \mathrm{E}$ & 10 & Continental Oil & Tribal 10-1 & 8,710 & 8,720 & Thermopolis & shale & 0.78 & 15 \\
\hline
\end{tabular}


Table 1. Vitrinite reflectance data and locations for cuttings samples from wells drilled for oil and gas exploration and production in the Wind River Basin, Wyoming. Map no. refers to sample location numbers shown on figure 2.-Continued

[API, well number assigned by American Petroleum Institute; Tnsp., township; Rng., range; Sec., section; Lith., lithology; \%Ro, vitrinite reflectance measurement; $n$, number of readings per sample; $\mathrm{nr}$, no reading. Depth is in feet.]

\begin{tabular}{|c|c|c|c|c|c|c|c|c|c|c|c|c|}
\hline $\begin{array}{c}\text { Map } \\
\text { no. }\end{array}$ & API & Tnsp. & Rng. & Sec. & Operator & Well & $\begin{array}{c}\text { Top } \\
\text { depth }\end{array}$ & $\begin{array}{c}\text { Bottom } \\
\text { depth }\end{array}$ & Formation & Lith. & $\% R_{0}$ & $\mathrm{n}$ \\
\hline 11 & 49013061630000 & $2 \mathrm{~N}$ & $2 W$ & 11 & Stanolind Oil \& Gas & 1 Yamba & 870 & 880 & Cody & shale & 0.89 & 10 \\
\hline 11 & 49013061630000 & $2 \mathrm{~N}$ & $2 W$ & 11 & Stanolind Oil \& Gas & 1 Yamba & 1,790 & 1,800 & Cody & shale & 0.97 & 16 \\
\hline 11 & 49013061630000 & $2 \mathrm{~N}$ & $2 W$ & 11 & Stanolind Oil \& Gas & 1 Yamba & 3,050 & 3,080 & Frontier & shale & 1.14 & 11 \\
\hline 11 & 49013061630000 & $2 \mathrm{~N}$ & $2 \mathrm{~W}$ & 11 & Stanolind Oil \& Gas & 1 Yamba & 3,230 & 3,240 & Mowry & shale & 0.99 & 11 \\
\hline 11 & 49013061630000 & $2 \mathrm{~N}$ & $2 W$ & 11 & Stanolind Oil \& Gas & 1 Yamba & 3,550 & 3,560 & Mowry & shale & 0.89 & 8 \\
\hline 11 & 49013061630000 & $2 \mathrm{~N}$ & $2 W$ & 11 & Stanolind Oil \& Gas & 1 Yamba & 3,660 & 3,730 & Thermopolis & shale & 1.10 & 14 \\
\hline 11 & 49013061630000 & $2 \mathrm{~N}$ & $2 W$ & 11 & Stanolind Oil \& Gas & 1 Yamba & 3,830 & 3,860 & Cloverly & shale & 1.28 & 12 \\
\hline 12 & 49013202830000 & $3 \mathrm{~N}$ & $1 \mathrm{~W}$ & 33 & Norris Oil & Tribal-McBride 1 & 3,230 & 3,260 & Frontier & shale & 0.29 & 1 \\
\hline 12 & 49013202830000 & $3 \mathrm{~N}$ & $1 \mathrm{~W}$ & 33 & Norris Oil & Tribal-McBride 1 & 3,440 & 3,470 & Mowry & shale & 0.54 & 11 \\
\hline 12 & 49013202830000 & $3 \mathrm{~N}$ & $1 \mathrm{~W}$ & 33 & Norris Oil & Tribal-McBride 1 & 3,650 & 3,670 & Mowry & shale & 0.51 & 14 \\
\hline 12 & 49013202830000 & $3 \mathrm{~N}$ & $1 \mathrm{~W}$ & 33 & Norris Oil & Tribal-McBride 1 & 3,850 & 3,870 & Thermopolis & shale & 0.51 & 11 \\
\hline 13 & 49013213430000 & $4 \mathrm{~N}$ & $3 \mathrm{~W}$ & 29 & Gulf Oil Company & Crowheart Butte SW 1-29-3D & 2,100 & 2,130 & Cody & shale & 0.67 & 12 \\
\hline 13 & 49013213430000 & $4 \mathrm{~N}$ & $3 \mathrm{~W}$ & 29 & Gulf Oil Company & Crowheart Butte SW 1-29-3D & 3,720 & 3,750 & Mowry & shale & 0.79 & 21 \\
\hline 13 & 49013213430000 & $4 \mathrm{~N}$ & $3 \mathrm{~W}$ & 29 & Gulf Oil Company & Crowheart Butte SW 1-29-3D & 4,110 & 4,140 & Mowry & shale & 0.99 & 19 \\
\hline 13 & 49013213430000 & $4 \mathrm{~N}$ & $3 \mathrm{~W}$ & 29 & Gulf Oil Company & Crowheart Butte SW 1-29-3D & 4,320 & 4,330 & Thermopolis & shale & 0.94 & 14 \\
\hline 14 & 49013203030000 & $4 \mathrm{~N}$ & $4 \mathrm{~W}$ & 35 & Stoltz \& Company & 1-35 Tribal & 600 & 610 & Mowry & shale & 1.10 & 8 \\
\hline 14 & 49013203030000 & $4 \mathrm{~N}$ & $4 \mathrm{~W}$ & 35 & Stoltz \& Company & 1-35 Tribal & 860 & 890 & Mowry & shale & 0.94 & 6 \\
\hline 14 & 49013203030000 & $4 \mathrm{~N}$ & $4 \mathrm{~W}$ & 35 & Stoltz \& Company & 1-35 Tribal & 1,050 & 1,060 & Thermopolis & shale & 0.89 & 13 \\
\hline 14 & 49013203030000 & $4 \mathrm{~N}$ & $4 W$ & 35 & Stoltz \& Company & 1-35 Tribal & 1,200 & 1,210 & Cloverly & shale & 1.06 & 13 \\
\hline
\end{tabular}


Table 1. Vitrinite reflectance data and locations for cuttings samples from wells drilled for oil and gas exploration and production in the Wind River Basin, Wyoming. Map no. refers to sample location numbers shown on figure 2.-Continued

[API, well number assigned by American Petroleum Institute; Tnsp., township; Rng., range; Sec., section; Lith., lithology; \%Ro, vitrinite reflectance measurement; $n$, number of readings per sample; $\mathrm{nr}$, no reading. Depth is in feet.]

\begin{tabular}{|c|c|c|c|c|c|c|c|c|c|c|c|c|}
\hline $\begin{array}{l}\text { Map } \\
\text { no. }\end{array}$ & API & Tnsp. & Rng. & Sec. & Operator & Well & $\begin{array}{c}\text { Top } \\
\text { depth }\end{array}$ & $\begin{array}{c}\text { Bottom } \\
\text { depth }\end{array}$ & Formation & Lith. & $\% R_{0}$ & $\mathrm{n}$ \\
\hline 15 & 49013065670000 & $5 \mathrm{~N}$ & $2 \mathrm{~W}$ & 6 & Empire State Oil & Tribal 1 & 1,780 & 1,800 & Cody & shale & 0.84 & 12 \\
\hline 15 & 49013065670000 & $5 \mathrm{~N}$ & $2 W$ & 6 & Empire State Oil & Tribal 1 & 2,800 & 2,820 & Mowry & shale & 0.66 & 13 \\
\hline 15 & 49013065670000 & $5 \mathrm{~N}$ & $2 \mathrm{~W}$ & 6 & Empire State Oil & Tribal 1 & 3,400 & 3,410 & Mowry & shale & 0.68 & 9 \\
\hline 15 & 49013065670000 & $5 \mathrm{~N}$ & $2 \mathrm{~W}$ & 6 & Empire State Oil & Tribal 1 & 3,650 & 3,660 & Thermopolis & shale & 0.64 & 7 \\
\hline 16 & 49013208510000 & $5 \mathrm{~N}$ & $5 \mathrm{~W}$ & 16 & Helmerich \& Payne & 1-16 Tribal & 440 & 460 & Mowry & shale & 1.01 & 9 \\
\hline 16 & 49013208510000 & $5 \mathrm{~N}$ & $5 \mathrm{~W}$ & 16 & Helmerich \& Payne & 1-16 Tribal & 750 & 760 & Mowry & shale & 0.85 & 10 \\
\hline 16 & 49013208510000 & $5 \mathrm{~N}$ & $5 \mathrm{~W}$ & 16 & Helmerich \& Payne & 1-16 Tribal & 900 & 910 & Thermopolis & shale & 0.84 & 12 \\
\hline 16 & 49013208510000 & $5 \mathrm{~N}$ & $5 \mathrm{~W}$ & 16 & Helmerich \& Payne & 1-16 Tribal & 1,100 & 1,110 & Cloverly & shale & 0.92 & 6 \\
\hline 17 & 49013067400000 & $6 \mathrm{~N}$ & $1 \mathrm{~W}$ & 4 & British-American Oil & Tribal 1 & 720 & 770 & Frontier & coal & 0.50 & 25 \\
\hline 17 & 49013067400000 & $6 \mathrm{~N}$ & $1 \mathrm{~W}$ & 4 & British-American Oil & Tribal 1 & 1,010 & 1,050 & Mowry & shale & 0.81 & 20 \\
\hline 17 & 49013067400000 & $6 \mathrm{~N}$ & $1 \mathrm{~W}$ & 4 & British-American Oil & Tribal 1 & 1,320 & 1,330 & Mowry & shale & 0.95 & 8 \\
\hline 17 & 49013067400000 & $6 \mathrm{~N}$ & $1 \mathrm{~W}$ & 4 & British-American Oil & Tribal 1 & 1,500 & 1,530 & Thermopolis & shale & 0.93 & 5 \\
\hline 18 & 49013206120000 & $1 \mathrm{~S}$ & $2 \mathrm{E}$ & 2 & Exxon Corporation & 1 Shoshone-Arapahoe 534 & 6,210 & 6,240 & Cody & shale & 0.47 & 17 \\
\hline 18 & 49013206120000 & $1 \mathrm{~S}$ & $2 \mathrm{E}$ & 2 & Exxon Corporation & 1 Shoshone-Arapahoe 534 & 7,600 & 7,610 & Cody & shale & 0.93 & 4 \\
\hline 18 & 49013206120000 & $1 \mathrm{~S}$ & $2 \mathrm{E}$ & 2 & Exxon Corporation & 1 Shoshone-Arapahoe 534 & 8,450 & 8,460 & Frontier & shale & 0.91 & 9 \\
\hline 18 & 49013206120000 & $1 \mathrm{~S}$ & $2 \mathrm{E}$ & 2 & Exxon Corporation & 1 Shoshone-Arapahoe 534 & 8,850 & 8,860 & Mowry & shale & 0.89 & 10 \\
\hline 18 & 49013206120000 & $1 S$ & $2 \mathrm{E}$ & 2 & Exxon Corporation & 1 Shoshone-Arapahoe 534 & 9,060 & 9,070 & Thermopolis & shale & 1.03 & 12 \\
\hline
\end{tabular}


Table 1. Vitrinite reflectance data and locations for cuttings samples from wells drilled for oil and gas exploration and production in the Wind River Basin, Wyoming. Map no. refers to sample location numbers shown on figure 2.-Continued

[API, well number assigned by American Petroleum Institute; Tnsp., township; Rng., range; Sec., section; Lith., lithology; \%Ro, vitrinite reflectance measurement; $n$, number of readings per sample; $\mathrm{nr}$, no reading. Depth is in feet.]

\begin{tabular}{|c|c|c|c|c|c|c|c|c|c|c|c|c|}
\hline $\begin{array}{l}\text { Map } \\
\text { no. }\end{array}$ & API & Tnsp. & Rng. & Sec. & Operator & Well & $\begin{array}{c}\text { Top } \\
\text { depth }\end{array}$ & $\begin{array}{c}\text { Bottom } \\
\text { depth }\end{array}$ & Formation & Lith. & $\% R_{0}$ & $\mathrm{n}$ \\
\hline 19 & 49013060040000 & $1 S$ & $5 \mathrm{E}$ & 1 & Continental Oil & 1 Tribal & 6,880 & 6,890 & Cody & shale & 0.85 & 10 \\
\hline 19 & 49013060040000 & $1 S$ & $5 \mathrm{E}$ & 1 & Continental Oil & 1 Tribal & 8,210 & 8,220 & Cody & shale & 0.86 & 16 \\
\hline 19 & 49013060040000 & $1 \mathrm{~S}$ & $5 \mathrm{E}$ & 1 & Continental Oil & 1 Tribal & 9,790 & 9,800 & Frontier & shale & 1.01 & 19 \\
\hline 19 & 49013060040000 & $1 S$ & $5 E$ & 1 & Continental Oil & 1 Tribal & 10,450 & 10,470 & Frontier & shale & 1.01 & 15 \\
\hline 19 & 49013060040000 & $1 S$ & $5 \mathrm{E}$ & 1 & Continental Oil & 1 Tribal & 10,850 & 10,860 & Mowry & shale & 1.19 & 13 \\
\hline 19 & 49013060040000 & $1 S$ & $5 \mathrm{E}$ & 1 & Continental Oil & 1 Tribal & 11,000 & 11,020 & Thermopolis & shale & 1.17 & 13 \\
\hline
\end{tabular}

